\title{
Photoelectron Spectroscopy at the Solid-Liquid Interface of Dye-Sensitized Solar Cells: Unique Experiments with the Solid-Liquid Interface Analysis System SoLiAS at BESSY
}

\author{
Konrad Schwanitz, Eric Mankel, Ralf Hunger, Thomas Mayer*, and Wolfram Jaegermann
}

\begin{abstract}
At the synchrotron BESSY we run the experimental station SoLiAS, dedicated to solid-liquid interface analysis with soft X-ray induced photoelectron spectroscopy (SXPS). SoLiAS allows wet chemically prepared surfaces to be transferred to the ultra high vacuum without contact with ambient air. In addition in situ (co)adsorption of volatile solvent species onto liquid nitrogen cooled samples is possible. SoLiAS proves to be very useful in analyzing the chemical and electronic structure at the solid-liquid interface of dye-sensitized solar cells. The standard dye Ru'(2,2'-bipyridil-4,4'-dicarboxylate) $)_{2}(\mathrm{NCS})_{2}$ was adsorbed from ethanol solution under clean $\mathrm{N}_{2}$ atmosphere in an UHV-integrated electrochemical cell (EC). The standard solvent acetonitrile was adsorbed in situ from the gas phase. For comparison also the nonpolar solvent benzene was adsorbed. Ex situ sintered nanocrystalline anatase substrates as well as in situ deposited polycrystalline $\mathrm{TiO}_{2}$ samples were used, which show a similar distribution of two types of occupied surface states. Distinct reversible changes occur in synchrotron-induced photoelectron valence band and core level spectra when the solvent acetonitrile is adsorbed to pristine and dye-covered $\mathrm{TiO}_{2}$ substrates. $\mathrm{TiO}_{2}$ surface states are quenched and the line width of the dye S2p emission decreases strongly. Based on the experimental results the alignment of the photovoltaic relevant electronic states and a model on the dye-solvent interaction can be deduced that points to the promotion of vectorial charge transfer by increased dye orientation due to solvation.
\end{abstract}

Keywords: Dye-sensitized solar cell · Photoelectron spectroscopy · Solid-liquid interface

\section{Introduction}

The photoelectrode of dye-sensitized solar cells consists of films of $\mathrm{TiO}_{2}$ in the anatase crystal structure stained by a monolayer of organic molecules sensitizing the wide gap semiconductor to visible light. Nanocrystalline (nc) and nanoporous $\mathrm{TiO}_{2}$ is

${ }^{\star}$ Correspondence: Dr. T. Mayer TU-Darmstadt

FB Material- und Geowissenschaften

FG Oberflächenforschung

Petersenstraße 23

D-64287 Darmstadt, Germany

E-Mail: mayerth@surface.tu-darmstadt.de used in order to increase the photoactive surface. ${ }^{[1]}$ The standard dye yielding the most efficient cells ${ }^{[2]}$ is $\mathrm{Ru}^{\mathrm{II}}\left(2,2^{\prime}\right.$-bipyridil4,4 '-dicarboxylate) ${ }_{2}(\mathrm{NCS})_{2} \quad(\mathrm{Ru}(\mathrm{N} 3))$. In the photovoltaic process, the photoexcited electron is transferred from the dye LUMO to the $\mathrm{TiO}_{2}$ conduction band while the hole in the HOMO is reduced in a reaction with a liquid electrolyte that is able to penetrate the pores of the nc-TiO, electrode. As standard electrolyte the redox couple $\mathrm{I}^{-} / \mathrm{I}_{3}{ }^{-}$dissolved in acetonitrile is used. Due to the nano-structured morphology, surfaces are of utmost importance as they are a source of intrinsic surface states due to under-coordination and reconstruction as well as of extrinsic surface states due to defects such as oxygen vacancies and adsorbates. In general, the density of the surface states is found in the fundamental gap of the semiconductor. Such gap states are electronically most active. They play a detrimental role in solar cells by decreasing the shunt resistance, thereby not only reducing the photocurrent but also the photovoltage. ${ }^{[3]}$ But recently $\mathrm{TiO}_{2}$ gap states have also been discussed to be beneficial in forming a transport band that improves the conductivity of the DSSC. ${ }^{[4]}$ Due to their importance in optoelectronic, catalytic and sensing applications $\mathrm{TiO}_{2}$ gap states have been analyzed with a number of methods e.g. electronically, ${ }^{[5]}$ with scanning probe methods ${ }^{[6]}$ and resonant photoelectron spectroscopy. ${ }^{[7]}$ Most of these studies have been performed on rutile single crystal surfaces because anatase single crystals are rare. The gap states have been correlated to a low binding energy shoulder of Ti2p core levels and therefore been assigned to occupied conduction band Ti3d states localized and stabilized on oxygen defect sites and by polaron formation. ${ }^{[8]}$

\section{Results and Discussion}

Using the linear extrapolation of the onset of the valence band (VB) photoelectron 
emission (Fig. 1), the valence band maximum (VBM) of pristine nanocrystalline (nc) $\mathrm{TiO}_{2}$ is found at $\mathrm{E}_{\mathrm{B}}=3.6 \mathrm{eV}$ binding energy (referenced to the Fermi level) while the fundamental gap is only $3.2 \mathrm{eV}$. In Fig. $1 \mathrm{VB}$ spectra of two samples prepared in situ by MOCVD using the single precursor titanium tetra isopropoxide (TTIP) as well as of two samples produced by ex situ sintering of a dispersion of $\mathrm{nc}-\mathrm{TiO}_{2}$ are displayed. In addition to the VB that consists mostly of $\mathrm{O} 2 \mathrm{p}$ orbitals, the $\mathrm{O} 2 \mathrm{~s}$ and Ti3p emissions are indicated in the spectra. Within the forbidden gap occupied states are found with a maximum at $\mathrm{E}_{\mathrm{B}}=1.3 \mathrm{eV}$ (Fig. 2). In addition states just below the Fermi level (JBF states) are found. These two types of states above the valence band are found on ex situ sintered as well as in situ deposited $\mathrm{TiO}_{2}$ films. In the course of acetonitrile adsorption the two types of states above the valence band maximum are quenched, but with different efficiency. The ratio of the emission of the states just below the Fermi level to the emission of the gap states at $1.3 \mathrm{eV}$ increases (Fig. 2). In order to eliminate damping due to adsorption, in Fig. 2 the spectra are displayed normalized to the Ti3p emission to evidence the quenching of the states above the VBM. The intensity of the occupied states above the VBM correlates with a low binding energy shoulder found in Ti2p core orbital spectra displayed in Fig. 3. In Fig. 3 a formal thickness of the adsorbed acetonitrile as calculated from the damping of the Ti3p emission is indicated. In addition to the Ti2p1/2
Ti2p3/2 duplet of the $\mathrm{Ti}^{4+}$ bulk emission a duplet shifted to lower binding energy is found that is addressed to a $\mathrm{Ti}^{3+}$ species. The relative intensity of the $\mathrm{Ti}^{3+}$ emission is reduced in the course of acetonitrile adsorption in parallel to the quenching of the states above the VBM. In Fig. 4 photon energy dependent spectra of the Ti2p core level are displayed. The $\mathrm{Ti}^{3+}$ emission is reduced with increasing photon energy due to an increase of the mean free path of the photoemitted electrons from approximately $0.6 \mathrm{~nm}$ at $600 \mathrm{eV}$ over $1.0 \mathrm{~nm}$ at $900 \mathrm{eV}$ to $1.4 \mathrm{~nm}$ at $1486 \mathrm{eV}$. Thus $\mathrm{Ti}^{3+}$ is clearly indicated as a surface species. The states above the valence band maximum are correlated with the $\mathrm{Ti}^{3+}$ emission and can be addressed to a surface $\mathrm{Ti}$ species with a $3 \mathrm{~d}^{1}$ configuration in contrast to $3 \mathrm{~d}^{0}$ of bulk Ti that forms the conduction band. Using the $\mathrm{Ti}^{3+} / \mathrm{Ti}^{4+}$ ratio we have calculated a density of around half a monolayer of surface states for pristine samples. ${ }^{[8]} \mathrm{A}$ similar distribution of two types of surface states has been observed with low intensity in resonant photoelectron spectroscopy on single anatase crystal (100) surfaces. ${ }^{[7]}$ While the gap states have been addressed to oxygen vacancies $\mathrm{V}_{\mathrm{O}}$, the origin of the states just below the Fermi level (JBF) is under debate.

In the most simple rigid band model (Fig. 5) we assign the JBF-states to occupied conduction band states of undisturbed stochiometric $\mathrm{TiO}_{2}$. But oxygen vacancies tend to agglomerate ${ }^{[9]}$ and titanium oxide can adopt many substochiometric phases and e.g. $\mathrm{Ti}_{2} \mathrm{O}_{3}$ is metallic. So the JBF states may be due to local agglomerations of oxygen vacancies or to a metallic $\mathrm{TiO}_{2-\mathrm{x}}$ phase.

The structure and a possible adsorption geometry of the $\mathrm{Ru}(\mathrm{N} 3)$ molecule are given in Fig. 6. With adsorption of the dye and coadsorption of acetonitrile the gap states are also quenched (Fig. 7). The highest occupied molecular orbital (HOMO) of the dye is found at $\mathrm{E}_{\mathrm{B}}=2.0 \mathrm{eV}$ before acetonitrile is added. Coadsorption of acetonitrile shifts the dye HOMO by $150 \mathrm{meV}$ to higher binding energy.

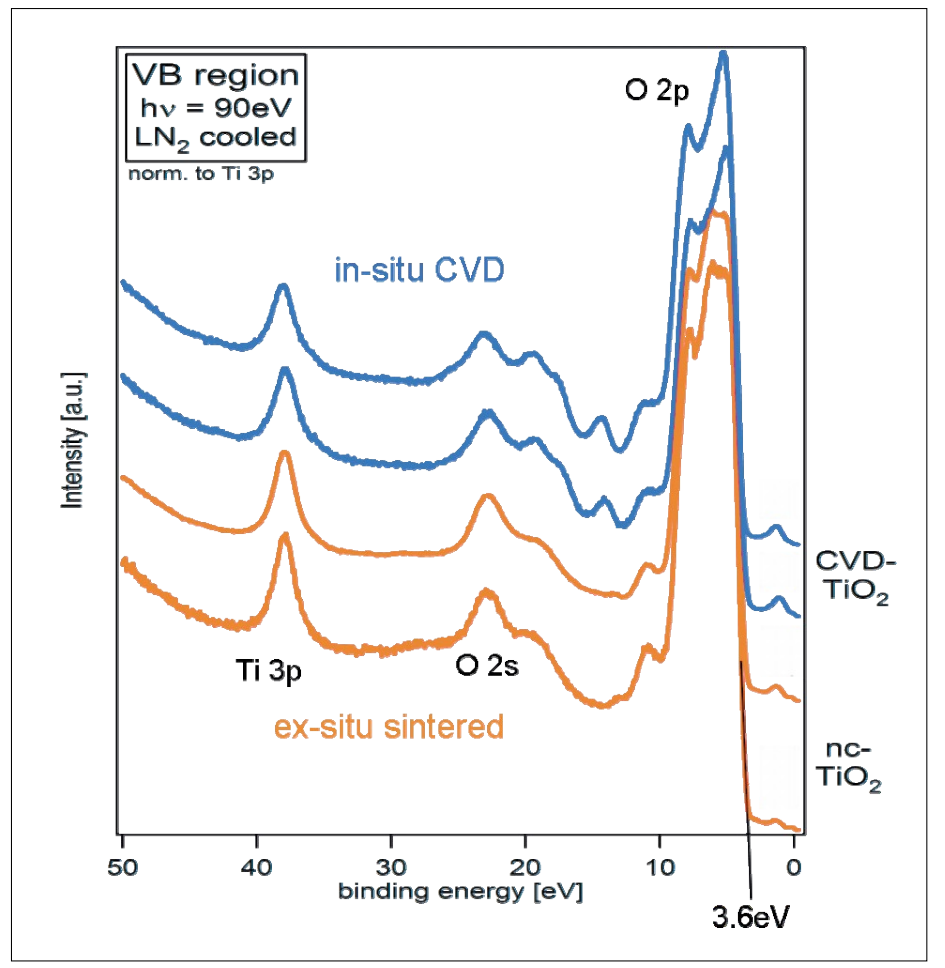

Fig. 1. SXPS spectra of two ex situ sintered and two in situ deposited $\mathrm{TiO}_{2}$ films

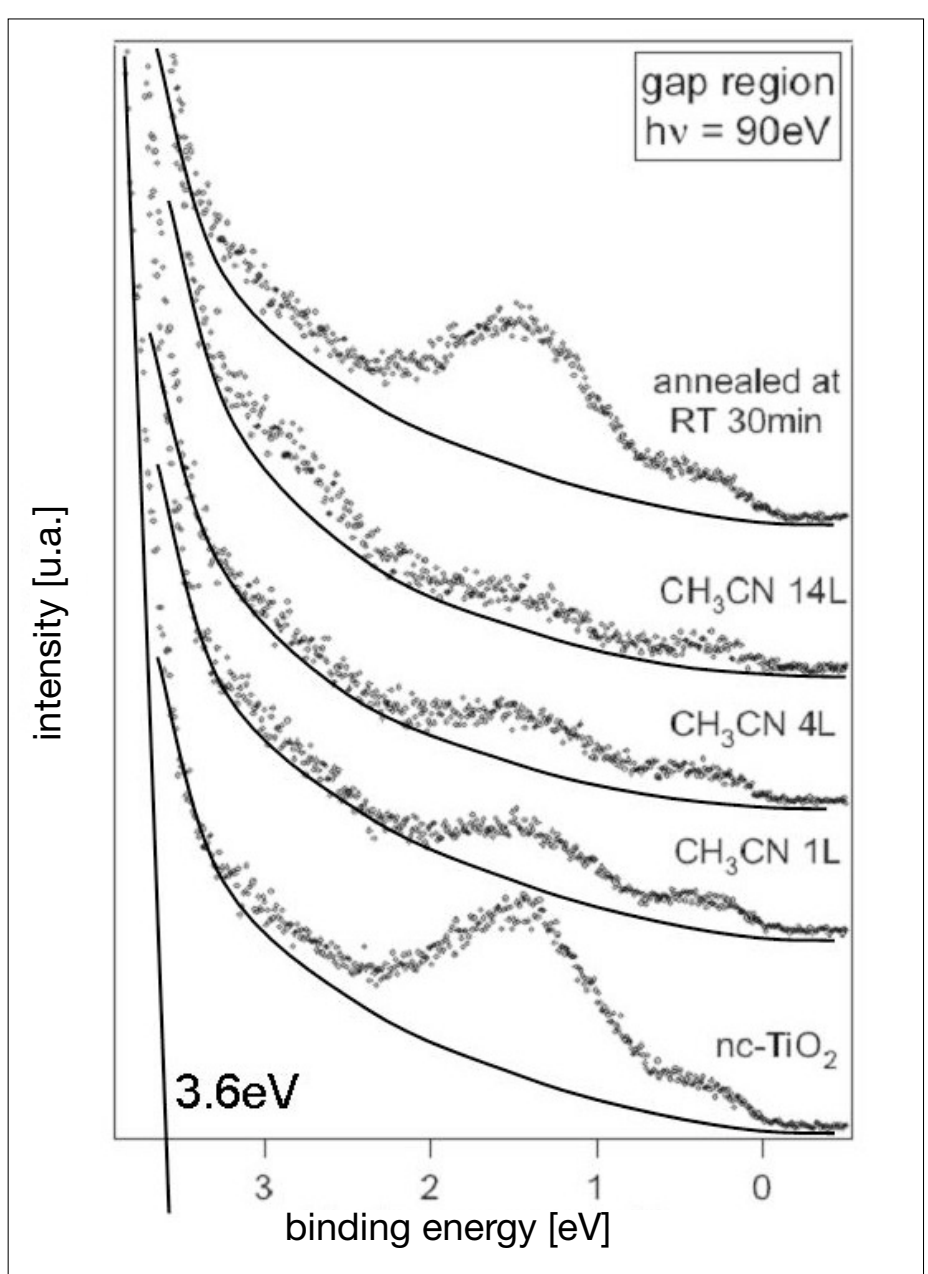

Fig. 2. SXPS spectra of the gap states of nanocrystalline anatase $\mathrm{TiO}_{2}$ in the course of acetonitrile adsorption and desorption. The spectra have been normalized in intensity to the Ti3p emission to eliminate damping due to adsorption. The states at $\mathrm{E}_{\mathrm{B}}=1.3 \mathrm{eV}$ are addressed to oxygen vacancies, while the origin of the states just below the Fermi level is under discussion. As a guide to the eye a background has been sketched to evidence the quenching of the states above the VBM. 


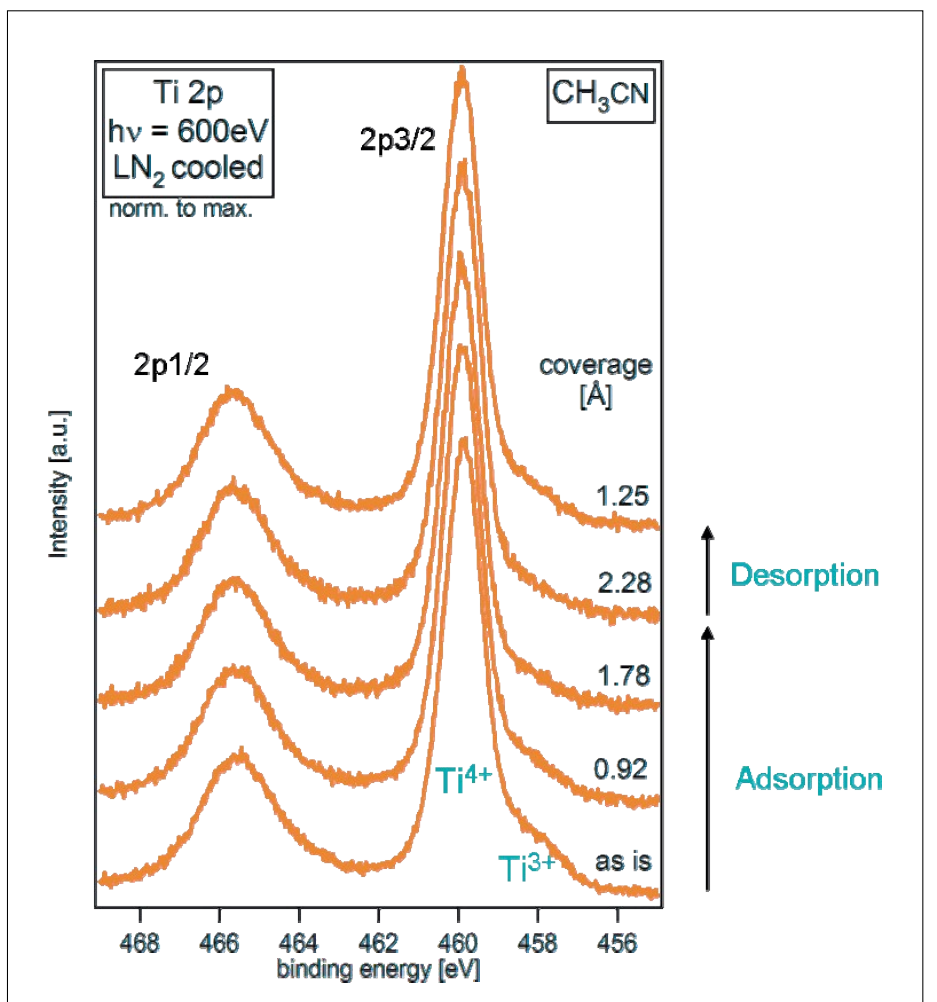

Fig. 3. SXPS of the Ti2p core level in the course of acetonitrile adsorption and desorption. The $\mathrm{Ti}^{3+}$ emission is quenched with increasing coverage and restored by desorption.

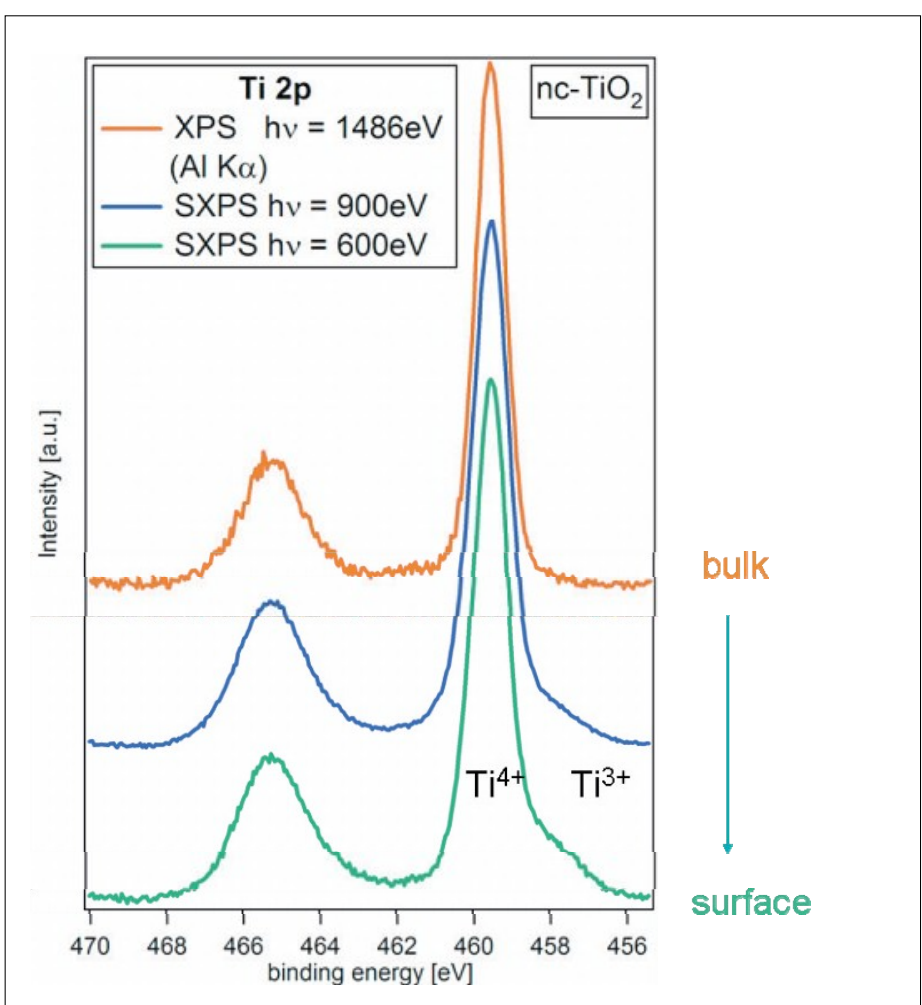

Fig. 4. The Ti2p core orbital of $\mathrm{nc}-\mathrm{TiO}_{2}$ measured with different surface sensitivity. From bottom to top the mean free path of the photo-excited electrons in $\mathrm{TiO}_{2}$ changes from $0.6 \mathrm{~nm}$ to $1.0 \mathrm{~nm}$ to $1.4 \mathrm{~nm}$. The $\mathrm{Ti}^{3+}$ component is clearly evidenced as a surface species.

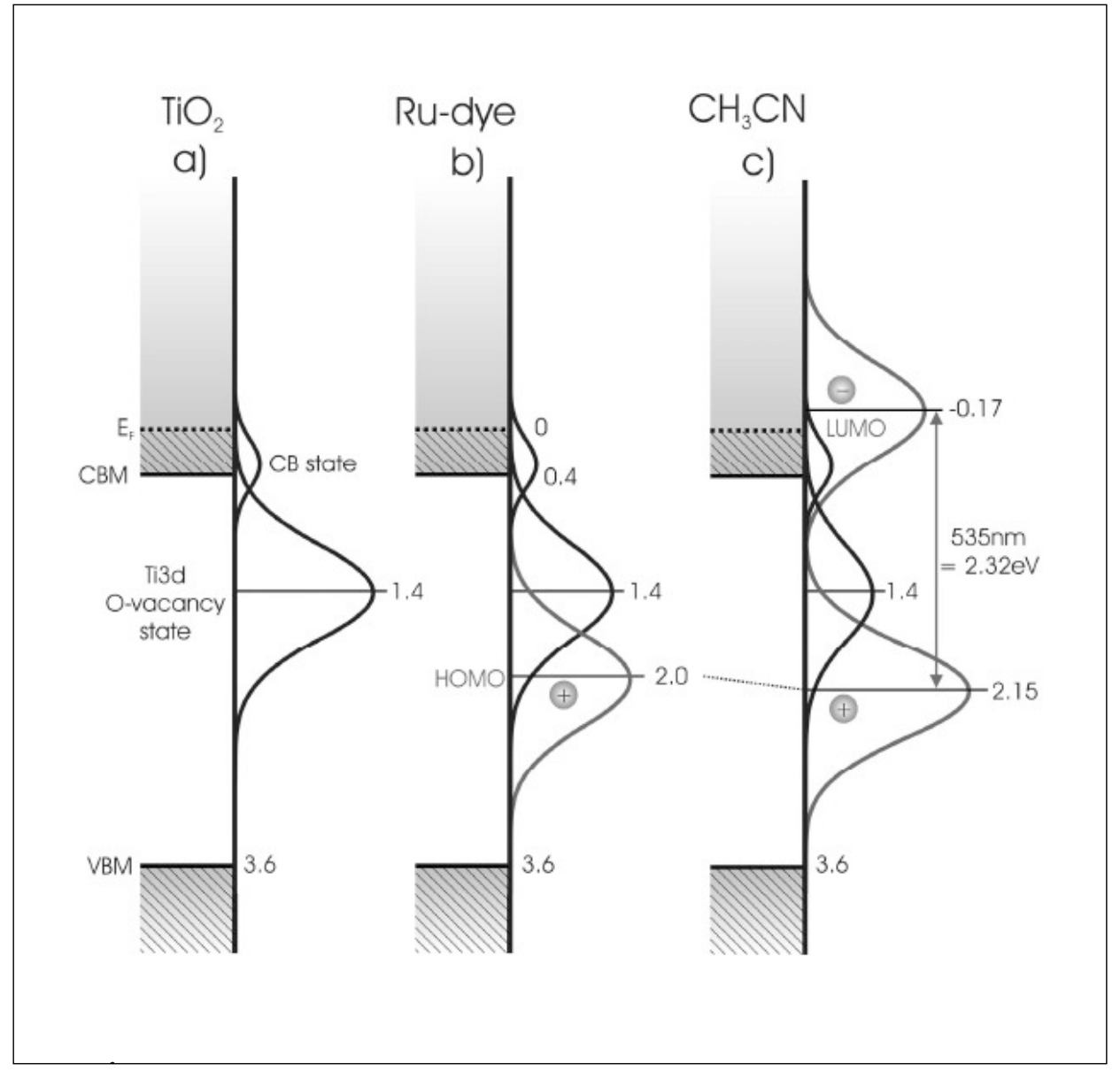

Fig. 5. Schematic of the photovoltaic-relevant valence states in the rigid band model: a) as prepared nanocrystalline $\mathrm{TiO}_{2}$ anatase film, b) after dye adsorption from ethanol solution with the HOMO position, c) after coadsorption of the solvent acetonitrile with the HOMO shifted by $150 \mathrm{meV}$ to higher $\mathrm{E}_{\mathrm{B}}$. Using the optical absorption maximum the $\mathrm{LUMO}$ is found $0.17 \mathrm{eV}$ above the Fermi level.

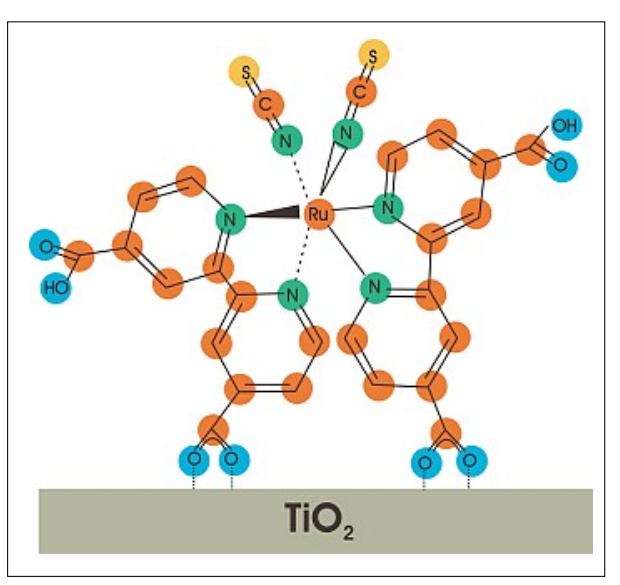

Fig. 6. Sketch of Ru'(2,2'-bipyridil-4,4'dicarboxylate) $)_{2}(\mathrm{NCS})_{2}(\mathrm{Ru}(\mathrm{N} 3))$ attached to the $\mathrm{TiO}_{2}$ surface by deprotonation. The HOMO is located mostly on the NCS groups and the LUMO on the bipyridil groups.

A schematic representation of the photovoltaic-relevant valence states as deduced in the simple rigid band model is displayed in Fig. 5. The measured HOMO position corresponds to the lowest energy hole-state created by photoemission of an electron i.e. the HOMO of the molecular cation. Except for a Franck Condon shift of approximately $0.1 \mathrm{eV}$ away from the Fermi level due to vibrational excitation in the photoemission process, this is the relevant energy position for the reduction of the photo hole by the redox electrolyte in the photovoltaic process. For the injection of the excited electron the 


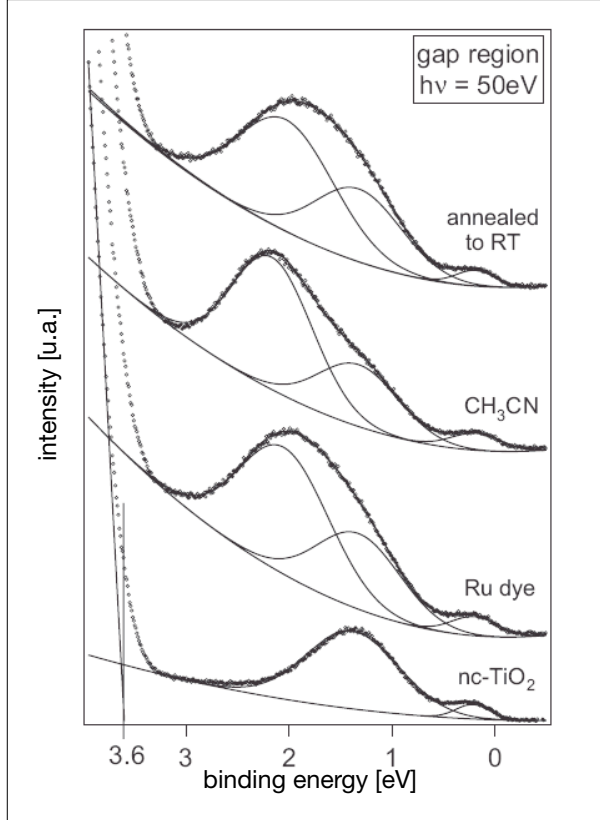

Fig. 7. SXPS spectra of the gap states of nanocrystalline anatase $\mathrm{TiO}_{2}$ in the course of $\mathrm{Ru}(\mathrm{N} 3)$-dye adsorption and acetonitrile coadsorption and desorption.

alignment of the LUMO to the conduction band edge is crucial. With photoemission we can detect occupied states only. Using the energy of the optical absorption maximum $(535 \mathrm{~nm}=2.32 \mathrm{eV})$, the LUMO is found approximately $0.17 \mathrm{eV}$ above the Fermi level.

For nc- $\mathrm{TiO}_{2}$ photo-electrodes of dyesensitized solarcells (DSSC) the distribution of surface states is of utmost importance as the transport of the injected photoelectron to the contact takes place along the surfaces of a number of crystallites. Surface states are electronically most active. In general they play a detrimental role in solar cells by decreasing the shunt resistance, thereby not

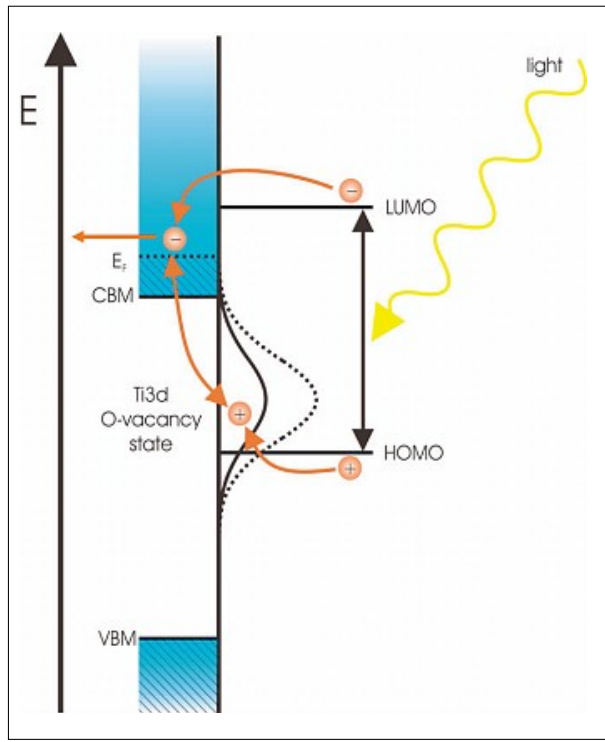

Fig. 8. Sketch of the different role the two types of surface states may play in the photovoltaic process of the DSSC only reducing the photocurrent but also the photovoltage. ${ }^{[3]}$ But recently $\mathrm{TiO}_{2}$ surface states have also been discussed to be beneficial in forming a transport band improving the conductivity of the DSSC. ${ }^{[4]}$ Because the surface states are quenched differently by the standard solvent acetonitrile, we conclude that the two types of surface states may play a different role in the DSSC as shown in Fig. 8. While the oxygen vacancies form a recombination channel for the photo excited electron hole pare, the states just below the Fermi level improve the conduction of the injected photoelectron to the metal contact. This hypothesis has to be confirmed in future by different measurement methods.

While the shape of the S2p line of the dye NCS group does not change when benzene is coadsorbed (Fig. 9b), the $\mathrm{S} 2 \mathrm{p}$ emission counter-intuitively sharpens upon $\mathrm{CH}_{3} \mathrm{CN}$ coadsorption (Fig. 9a). In a simple model as shown in Fig. 10a the polar solvent acetonitrile is able to penetrate the dye layer and the interaction of the dye NCS groups with the $\mathrm{TiO}_{2}$ surface and with neighboring dye molecules, the cause of $S 2 p$ broadening, are lifted due to dye solvation. The $\mathrm{S} 2 \mathrm{p}$ intensity development in the course of $\mathrm{CH}_{3} \mathrm{CN}$ adsorption suggests reorientation of the dye molecules pointing their NCS groups away from the surface towards the electrolyte. On the other hand nonpolar benzene just covers the dye layer (Fig. 10b). Since the HOMO is located mostly on the NCS groups and the LUMO mostly on the bipyridil groups, the orientation of the molecule is important for obtaining charge transfer of the excited electron from the dye LUMO to the $\mathrm{TiO}^{2}$ conduction band and the hole from the dye HOMO to the electrolyte. Solvation and orientation of the dye is important for obtaining the photovoltaic crucial vectorial charge transfer. The demonstrated specific action of acetonitrile on the dye may be considered as the reason why acetonitile has become the standard solvent.

\section{Summary}

Synchrotron-induced photoelectron spectroscopy SXPS proves to be very useful in analyzing the chemical and electronic structure at the solid-liquid interface of dyesensitized solar cells. Ex situ sintered and in situ deposited samples show two types of surface states. Distinct reversible changes occur in SXPS valence band and core level spectra when the solvent acetonitrile is adsorbed to pristine and dye-covered $\mathrm{TiO}_{2}$ substrates. Type 1 surface states at a binding energy of $1.3 \mathrm{eV}$ below the Fermi level are addressed to occupied Ti3d orbitals due to oxygen vacancies $\mathrm{V}_{\mathrm{O}}$. The origin of type 2 states at binding energies just below the Fermi level has been discussed in the most simple rigid band model as occupied con- duction band states. But also agglomeration of $\mathrm{V}_{\mathrm{O}}$ and formation of a substoichiometric metallic $\mathrm{TiO}_{2-\mathrm{x}}$ surface phase was considered. Since the standard solvent acetonitrile quenches type 1 surface states strongly and type 2 weakly we conclude that the $\mathrm{V}_{\mathrm{O}}$ states form a recombination path detrimental for the cell efficiency while type 2 surface states are beneficial in increasing conduction of the injected photoelectron to the metal contact. Coadsorption of acetonitrile to the dye layer is accompanied by peculiar changes of the spectra. The binding energy of the HOMO is increased by $150 \mathrm{meV}$ and the line width of the dye S2p emission is decreased while its intensity is restored. A model of the dye-solvent interaction has been deduced. In this model the dye molecules become insulated from each other and from the substrate by solvation and the dye NCS group becomes oriented towards the electrolyte. Since the photo-hole has to be transferred from the HOMO located on the NCS group to the electrolyte the vectorial charge transfer is promoted. Based on the experimental results the alignment of the photovoltaic relevant electronic states has been deduced.

\section{Experimental Section}

Experiments were conducted on our endstation SoLiAS (solid-liquid interface analysis system) at the undulator beamline U49 at the synchrotron light source BESSY. SoLiAS provides a ultra high vacuum (UHV) base pressure in the range of $10^{-10}$ mbar. Details of SoLiAS and typical applications may be found in the literature. ${ }^{[10,11]}$ Two types of samples have been prepared either ex situ by sintering at $450^{\circ} \mathrm{C}$ a colloid of particles of around $20 \mathrm{~nm}$ (Solaronix SA) or in situ by metal organic chemical vapor deposition (MOCVD) decomposing the precursor titanium tetraisopropoxide (TTIP 99.995\% Alfa Aesar) heated to $80{ }^{\circ} \mathrm{C}$. The deposition was performed at a pressure of $10^{-5}$ mbar for $2.5 \mathrm{~h}$ onto the native oxide surface of a $\mathrm{Si}$ (111) wafer at $450{ }^{\circ} \mathrm{C}$. To adsorb the standard dye $\mathrm{Ru}^{\mathrm{II}}\left(2,2^{\prime}\right.$-bipyridil-4,4'-dicarboxylate) ${ }_{2}$ (NCS) ${ }_{2}$ dissolved in ethanol (Solaronix S.A. Switzerland) an UHV-integrated electrochemical glass cell was used that is purged with a continuous flow of pure argon and allows a direct transfer to an UHV buffer chamber. The dye was adsorbed for $3 \mathrm{~min}$. By rinsing with pure ethanol left over dye molecules not anchored to $\mathrm{TiO}_{2}$ were washed off. For condensation of the solvent acetonitrile $(99.8 \%$ Alfa Aesar) the sample was cooled by contact to a liquid nitrogen $\left(\mathrm{LN}_{2}\right)$ reservoir on the UHV manipulator that allows the sample to be moved, actively cooled, between an adsorption chamber and the analysis chamber. All spectra were taken at approximately $\mathrm{LN}_{2}$ temperature. The binding energy scale 


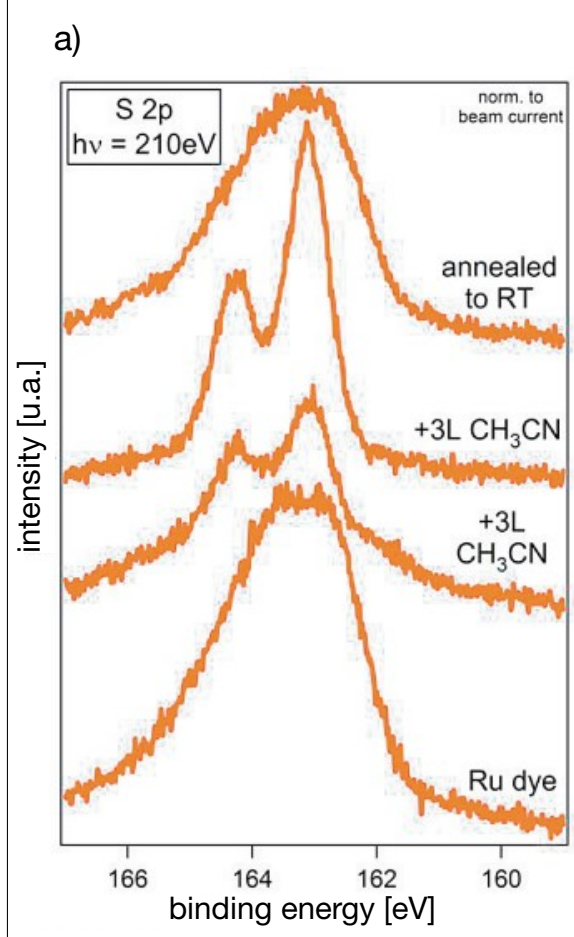

b)

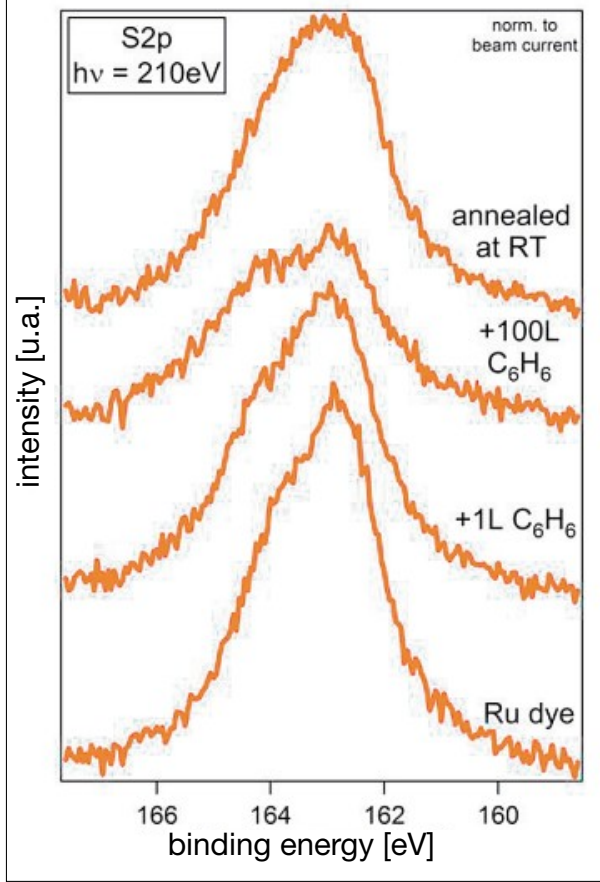

Fig. 9. a) Ru(N3)-dye S2p level in the course of acetonitrile and $b$ ) benzene coadsorption and desorption. The changes of the line shape and intsity in a) are interpreted as solvation and orientation of the dye.

of the spectra was referenced to the Fermi edge of a metallic probe.

\section{Acknowledgement}

Funding of the project by DFG under contract JA $859 / 3-3$, of SoLiAS by BMBF under project no. $05 \mathrm{KSIRD1} / 0$, and of BESSY beamtime by BMBF under no. $05 \mathrm{ES} 3 \mathrm{XBA} / 5$ is gratefully acknowledged.

Received: October 29, 2007 a)

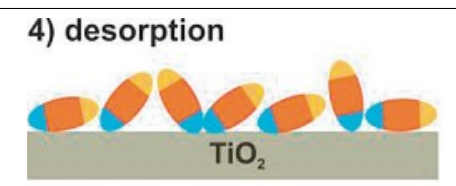

3) more $\mathrm{CH}_{3} \mathrm{CN}$

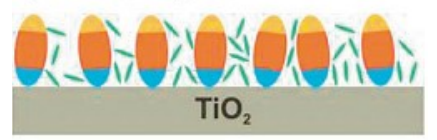

2) $\mathrm{CH}_{3} \mathrm{CN}$ coadsorption

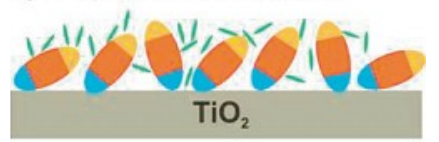

1) Ru-dye adsorption

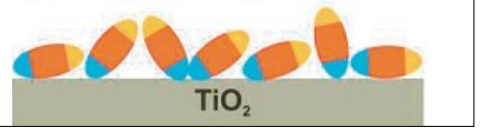

b)

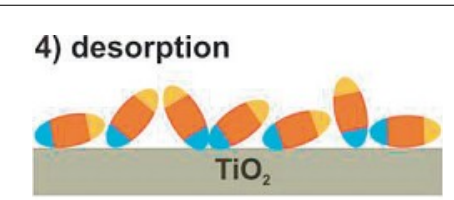

3) more $\mathrm{C}_{6} \mathrm{H}_{6}$

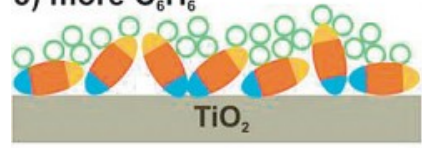

2) $\mathrm{C}_{6} \mathrm{H}_{6}$ adsorption

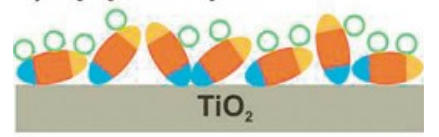

1) Ru-dye adsorption

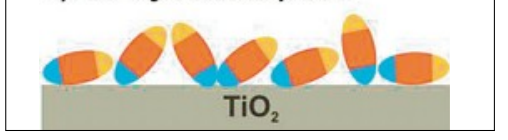

Fig. 10. a) Cartoon of the Ru(N3)-dye/solvent interaction in the course of acetonitrile and b) benzene coadsorption and desorption

[1] B. O’Regan, M. Grätzel, Nature 1991, $353,737$.

[2] M. K. Nazeeruddin, A. Kay, I. Rodicio, R. Humpbry-Baker, E. Miller, P. Liska, N. Vlachopoulos, M. Grätzel, J. Am. Chem. Soc. 1993, 115, 6382.

[3] A. N. M. Green, E. Palomares, S. A. Haque, J. M. Kroon, J. R. Durrant, $J$. Phys. Chem. B 2005, 109, 12525.

[4] B. A. Gregg, Coord. Chem. Rev. 2004, 248,1215 .
[5] N. Kopidakis, K. D. Benkstein, J. van de Lagemaat, A. J. Frank, Q. Yuan, E. A. Schiff, Phys. Rev. B 2006, 73, 045326.

[6] O. Dulub, C. Di Valentin, A. Selloni, U. Diebold, Surf. Sci. 2006, 600, 4407.

[7] A. G. Thomas, W. R. Flavell, A. K. Mallick, A. R. Kumarasinghe, D. Tsoutsou, N. Khan, C. Chatwin, S. Rayner, G. C. Smith, R. L. Stockbauer, S. Warren, T. K. Johal, S. Patel, D. Holland, A. Taleb, F. Wiame, Phys. Rev. B 2007, 75, 035105.

[8] K. Schwanitz, U. Weiler, R. Hunger, T. Mayer, W. Jaegermann, J. Phys. Chem. C 2007, 111, 849 .

[9] O. Dulub, M. Batzill, S. Solovev, E. Loginova, A. Alchagirov, T. E. Madey, U. Diebold, Science 2007, 317, 1052.

[10] T. Mayer, M. Lebedev, R. Hunger, W. Jaegermann, Appl. Surf. Sci. 2005, 252, 31.

[11] W. Jaegermann, T. Mayer, Solar Energy Materials and Solar Cells 2004, 83, 371. 